\title{
"Drowning Brain in a Pool of CSF" - A Rare Complication of Periencephalic Subdural Panhygroma following Removal of a Posterior Fossa Tumor
}

\author{
Bikash Ranjan Behera ${ }^{1}$ Ram Chandra Deo ${ }^{1} \quad$ Sanjib Mishra ${ }^{1} \quad$ Jyotirmayee Biswal ${ }^{2}$ Deepak Das ${ }^{1}$
}

${ }^{1}$ Department of Neurosurgery, SCB Medical College, Cuttack, India

Address for correspondence Bikash Ranjan Behera, MS, Qr/No-

2Peerless Hospital, Kolkata, West Bengal, India B/L-29, VSS Nagar, PO Sahid Nagar, Bhubaneswar, Odisha 751007 , India (e-mail: drtinku007@gmail.com).

Indian J Neurosurg 2018;7:223-226

\begin{abstract}
Posterior fossa tumors are commonly encountered in pediatric age group patients. Most of these tumors present with features of hydrocephalus in the child. Conventionally, these cases are managed by suboccipital craniotomy with decompression of the tumor mass to establish the free flow of cerebrospinal fluid (CSF) across fourth ventricle and aqueduct of Sylvius. Following resection of posterior fossa tumor, appearance

Keywords

- posterior fossa tumor resection

- periencephalic subdural panhygroma

- ventriculoperitoneal shunt of subdural hygroma is a rare phenomenon.

Though few cases of subdural hygroma are reported in literature following foramen magnum decompression in Chiari's malformation, their appearance following posterior fossa tumor resection is alien to medical literature and limited to only two case reports. Here the authors present a patient with periencephalic subdural panhygroma (PSP) following posterior fossa tumor resection who was successfully treated with a ventriculoperitoneal shunt (VPS) to accomplish a symptomatic and radiologic remission.
\end{abstract}

\section{Introduction}

Periencephalic subdural panhygroma (PSP), following posterior fossa tumor resection (PFTR) is an unusual complication. Post-PFTR clinical improvement, followed by gradual onset nonspecific symptoms of raised intracranial pressure (ICP), points out a possibility toward this unusual complication.

Though the exact mechanism of formation of PSP is unknown, the authors speculate the conversion of previously persisting internal hydrocephalus to postoperative external hydrocephalus in a noncompliant brain due to formation of a huge subdural space freely communicating with major cisterns.

Most of the postoperative subdural hygroma reports are confined to foramen magnum decompression in Chiari's malformations. ${ }^{1,2}$ Here the authors describe a case of subdural hygroma involving both supra- and infratentorial space and proposed a nomenclature of "periencephalic subdural panhygroma" (PSP) that is first of its kind in present-day medical literature.

Because of the paucity of this complication, its management is not standardized and purely driven by clinician's choice. ${ }^{3}$ Here the authors propose a suitable nomenclature with possible mechanism of formation and a rational treatment for this rare complication.

\section{Case Description}

A 1.5 -year-old male child weighing $5 \mathrm{~kg}$ was admitted to the department with complaints of irritability, vomiting, decrease in the movements of all four limbs, and multiple episodes of seizure for last 4 months. The baby had normal antenatal, natal, and postnatal history with normal-term vaginal delivery. received

August 6, 2016

accepted

February 7, 2017

published online

October 12, 2017
DOI https://doi.org/

10.1055/s-0037-1602751.

ISSN 2277-954X.
C2018 Neurological Surgeons'

Society of India
License terms

$($ () (1) $\Theta \circledast$ 
On examination, the baby was irritable having emaciated and deprived look ( - Fig. 1A). On physical examination the head circumference was $54 \mathrm{~cm}$ with open and bulged anterior fontanelle. Head control was not achieved. Menace reflex was present. Extraocular movements were full with normal light reflex in both the eyes with no apparent facial weakness. There was no nasal regurgitation of food or nasal intonation of crying sound. On examination of motor system, all four limbs were having power of grade 5/5 with intermittent extensor posturing of lower limbs. Bowel and bladder habit was apparently normal.

Magnetic resonance imaging (MRI) of the brain revealed a well-circumscribed T1-hypointense, T2-hyperintense, with peripheral enhancing cystic mass of size $65 \times 58 \times 51 \mathrm{cc}$ in the midline posterior fossa region compressing the fourth ventricle and brainstem with gross obstructive hydrocephalus and thinned-out cerebral/ cerebellar parenchyma (-Fig. 1B-D).

The patient was planned for suboccipital craniotomy with a telovelar approach for excision of this posterior fossa mass. A pinkish white, cystic, well-capsulated, moderately vascular mass was discovered. After complete excision of the mass, aqueduct of Sylvius was left well wide open with pulsating CSF slowly filling the roomy postoperative posterior fossa cavity (-Fig. 1E). Dura closed in watertight manner to prevent CSF leak.

In postoperative period, the patient showed significant clinical improvement and put on oral diet. On ninth postoperative day, the child became drowsy multiple episodes of vomiting with one episode of seizure with extensor posturing. He was put on intravenous fluids with antiepileptics and sent for a noncontrast computed tomography (NCCT) scan of the brain in emergency basis, which revealed an intercommunicating subdural hygroma spanning both supra- and infratentorial compartment of the brain with a wide and patent aqueduct (-Fig. 1F). This unusual phenomenon tempted the authors to propose a new term "periencephalic subdural panhygroma" (PSP). To relieve the raised ICP, a ventriculoperitoneal shunt (VPS) was done in emergency basis. Post-VPS, a significant improvement in sensorium was observed in the child. Even though the clinical status of the child improved, NCCT of the brain still showed presence of PSP with shunt in situ
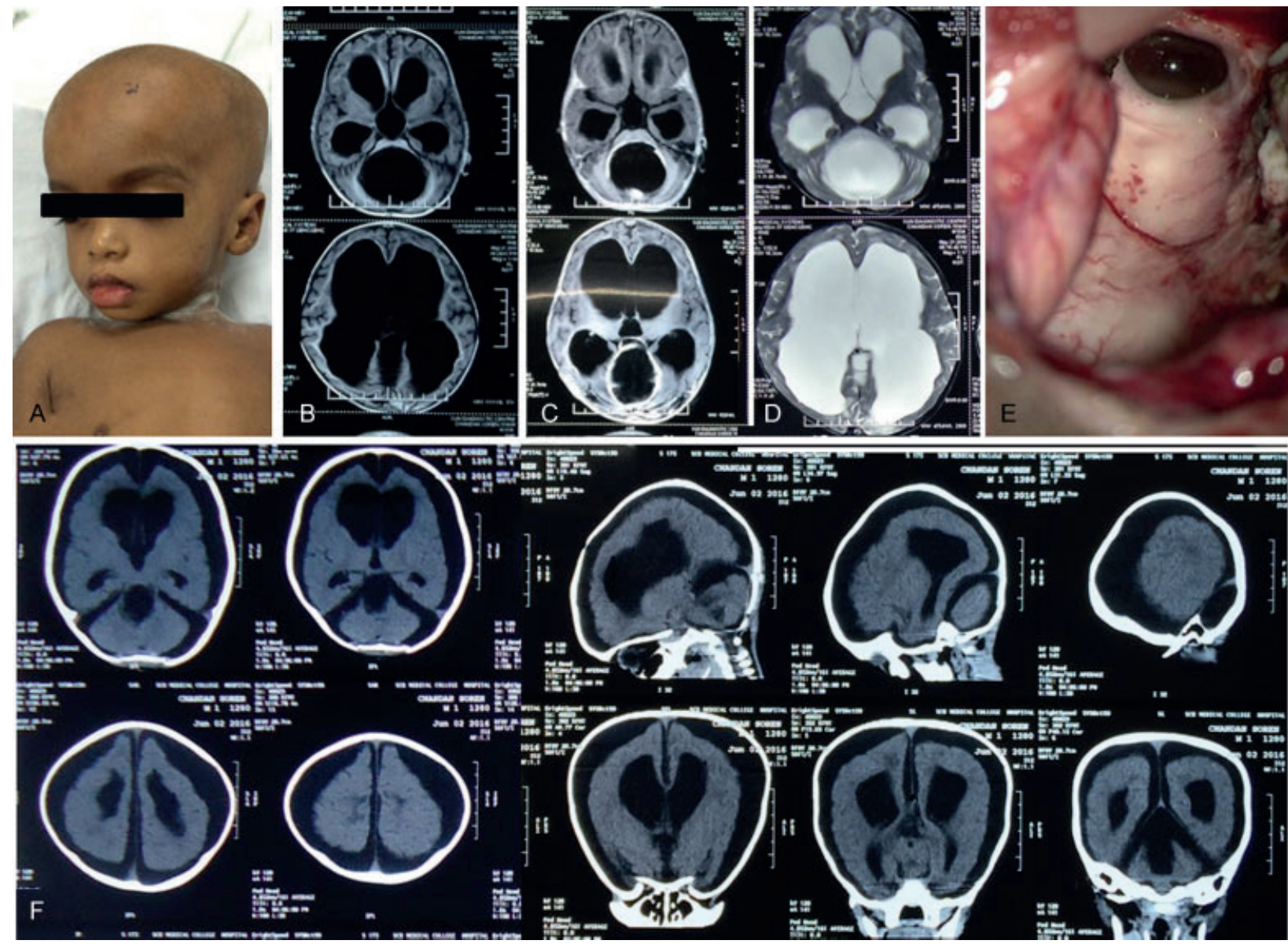

Fig. 1 (A) Preoperative picture of the child. (B) MRI of the brain T1 sequence showing hypointense cystic mass in the midline cerebellar region compressing fourth ventricle with gross hydrocephalus. (C) MRI of the brain T1 contrast, featuring a midline cerebellar peripheral contrast-enhancing cystic mass compressing fourth ventricle. (D) MRI of the brain T2 sequence showing cystic midline cerebellar mass isointense with CSF compressing fourth ventricle. (E) Post-tumor resection view of roomy posterior fossa with visible aqueduct of Sylvius with free-flowing CSF. (F) Postoperative NCCT of the brain showing both supra- and infratentorial subdural hygroma with dilated ventricles and brain parenchyma being compressed both intra- and extra-axially. CSF, cerebrospinal fluid; MRI, magnetic resonance imaging; NCCT, noncontrast computed tomography. 


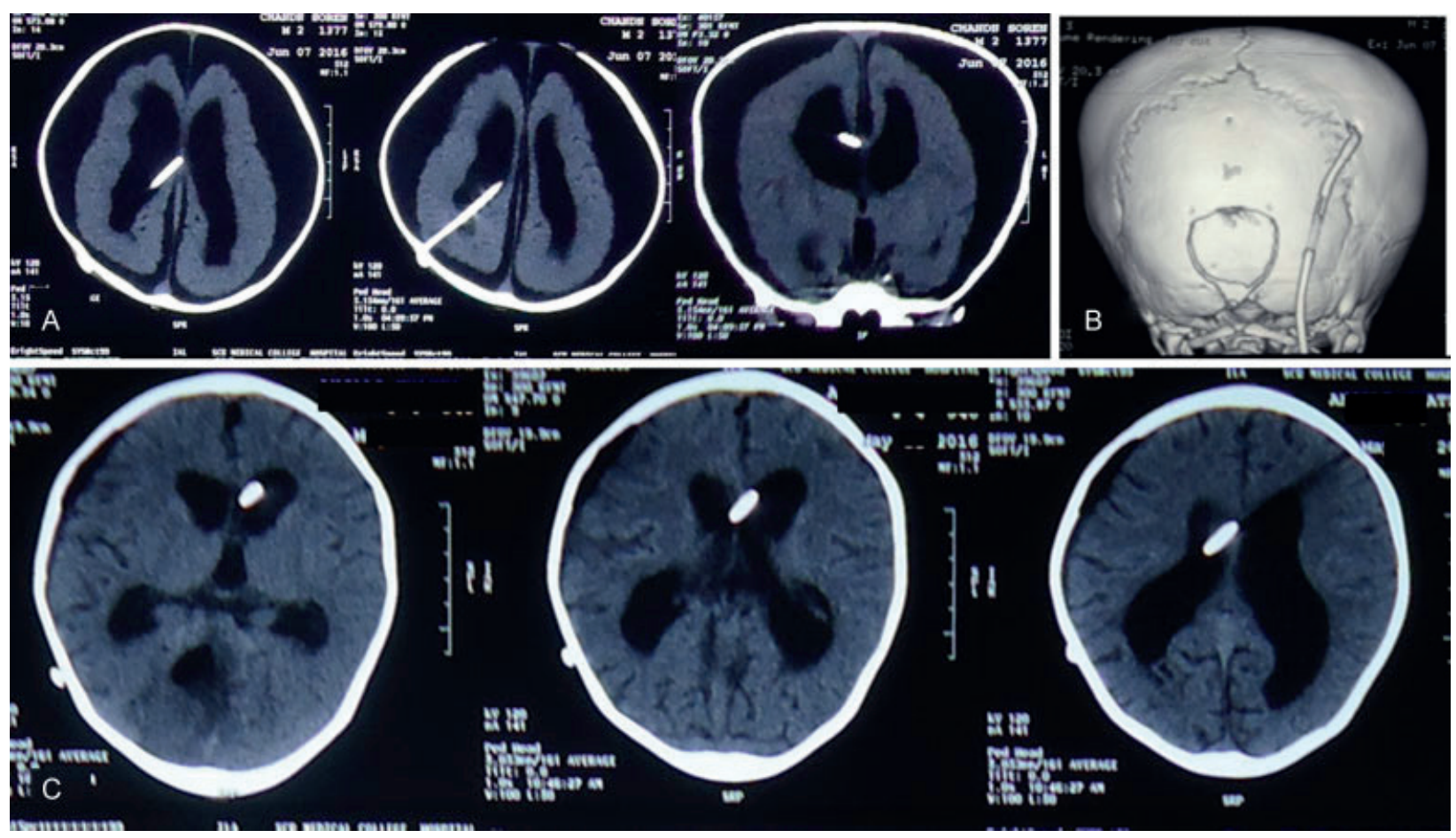

Fig. 2 (A) Post-ventriculoperitoneal shunt NCCT of the brain showing presence of subdural hygroma with shunt in situ. (B) Three-dimensional reconstruction of skull bone showing suboccipital craniotomy defect with right-sided VP shunt in situ. (C) Three-month follow-up NCCT of the brain (axial view) showing complete resolution of subdural hygroma with shunt in situ. NCCT, noncontrast computed tomography; VP, ventriculoperitoneal.

( Fig. 2A). After 1 week the child was discharged with oral medication. The histopathology of the tumor came out to be "hemangioblastoma."

In the first follow-up after 3 months, the child showed significant weight gain with encouraging social smile. Threemonth postoperative NCCT of the brain showed almost completely resolved PSP with shunt in situ ( - Fig. 2C).

\section{Discussion}

Subdural hygroma was first reported in 1916 by Payr, ${ }^{4}$ which he described as "meningitis serosa traumatica" that was later redescribed as "subdural hygroma" by Dandy in 1932.

Infantile subdural hygroma of surgical interest represents an extremely heterogenous group of pathology with various etiologic factors such as pneumococcal leptomeningeal infections, ${ }^{5}$ late complications of traumatic brain injury, ${ }^{5}$ and several neurosurgical procedures. ${ }^{6}$

Subdural hygroma as a complication of post-foramen magnum decompression (FMD) for Chiari's malformation is a known fact and supported by few medical literatures. ${ }^{1,2,7}$ The exact pathophysiology of hygromas post-FMD where durotomy was not performed is unknown, although it is supposed to be contributed by few microscopic breeches during procedure. ${ }^{2}$

Subdural hygroma in infants mostly presents with features of raised ICP, such as irritability, vomiting, altered sensorium, seizure, and focal neurological deficit due to compressive effect on brain parenchyma. ${ }^{8-10}$

The mechanism of formation of postoperative subdural hygroma is not fully understood. Here, the authors propose the possible mechanism of periencephalic subdural panhygroma in an infant with postoperative posterior fossa tumor resection as follows:

"Long duration hydrocephalus makes the infantile brain noncompliant. The extra space achieved in this noncompliant infantile brain after resection of a huge tumor mass is not compensated by proper enlargement of brain parenchyma; rather it encourages the formation of a potential subdural space around the brain by the ball-valve mechanism of the arachnoid tear where the CSF enters into the subdural space but can't escape, leading to formation of a PSP in order to maintain the pressure-volume homeostasis within the rigid skull bone. Later, there is a complete communication between intra- and extra-axial compartments with free-flowing CSF having no actual pressure difference between two compartments."

Several management protocols of subdural hygroma are described in literature (-Table $\mathbf{1}$ ), each having specific advantages and disadvantages. ${ }^{9}$ Here, contrary to their expectation, the authors observed that CSF coming out just after durotomy was not in pressure. Therefore, the authors preferred a VPS to a subdural-peritoneal shunt for long-term management. This patient with VPS exhibited good clinical and radiologic outcome in follow-up period.

\section{Conclusion}

PSP following resection of a posterior fossa tumor can be rightly described as "a drowning brain in a pool of CSF." VPS is an effective mode of treatment for these cases. Paucity of similar complications in medical literature makes this report an interesting read. 
Table 1 Previous cases of subdural hygroma following posterior fossa surgery

\begin{tabular}{|l|l|l|l|l|l|}
\hline Authors & Year & Journal & No & Pathology & Management \\
\hline Albuquerque et al $^{11}$ & 1997 & JNS & 5 & Arachnoid cyst rupture & $\begin{array}{l}\text { External subdural drain, sub- } \\
\text { dural peritoneal shunt }\end{array}$ \\
\hline${\text { Ranjan et } \text { al }^{7}}$ & 1996 & BJN & 1 & Chiari's malformation & Conservative \\
\hline Marshman et al $^{12}$ & 2005 & JNS & 2 & Chiari's malformation & VPS, conservative \\
\hline Stavrinous et al $^{13}$ & 2008 & BJN & 1 & Tumor hemangioblastoma & Burr hole + needle aspiration \\
\hline Filis et al $^{14}$ & 2009 & Paed NS & 1 & Chiari's malformation & Conservative, acetazolamide \\
\hline Suzuki et al ${ }^{15}$ & 2011 & Neurol Med Chir (Japan) & 1 & Chiari's malformation & Arachnoid ectomy \\
\hline Bahuleyan et al $^{16}$ & 2011 & JNS & 2 & Chiari's malformation & Burr hole/VPS \\
\hline Oomman et al & 2014 & Arch Int Surg & 2 & Posterior fossa tumor & VPS \\
\hline
\end{tabular}

Abbreviation: VPS, ventriculoperitoneal shunt.

\section{References}

1 Elton S, Tubbs RS, Wellons JC III, Blount JP, Grabb PA, Oakes WJ. Acute hydrocephalus following a Chiari I decompression. Pediatr Neurosurg 2002;36(02):101-104

2 Bahl A, Murphy M, Thomas N, Gullan R. Management of infratentorial subdural hygroma complicating foramen magnum decompression: a report of three cases. Acta Neurochir (Wien) 2011;153(05):1123-1128

3 Oomman A, Rajalingam V. Subdural hygroma following posterior fossa tumor resection. Arch Int Surg 2014;4:193-199

4 Payr E. Meningitis serosa bei und nach Schädelverletzungen (traumatica) Med Klin 1916;12:841-846

5 Caldarelli M, Di Rocco C, Romani R. Surgical treatment of chronic subdural hygromas in infants and children. Acta Neurochir (Wien) 2002;144(06):581-588, discussion 588

6 Mizoi K, Takaku A, Suzuki J. Subdural effusion following radical surgery for chiasmal region tumors in children. Report of 4 cases. Childs Brain 1981;8(04):307-315

7 Ranjan A, Cast IP. Symptomatic subdural hygroma as a complication of foramen magnum decompression for hindbrain herniation (Arnold-Chiari deformity) Br J Neurosurg 1996;10(03):301-303

8 Morota N, Sakamoto K, Kobayashi N, Kitazawa K, Kobayashi S. Infantile subdural fluid collection: diagnosis and postoperative course. Childs Nerv Syst 1995;11(08):459-466

9 Forman PM, Chipps BE, Meyer GA. Managing chronic subdural hematomas and effusions in infants: a continuing dilemma. Tex Med 1974;70(04):62-66
10 Litofsky NS, Raffel C, McComb JG. Management of symptomatic chronic extra-axial fluid collections in pediatric patients. Neurosurgery 1992;31(03):445-450

11 Albuquerque FC, Giannotta SL. Arachnoid cyst rupture producing subdural hygroma and intracranial hypertension: case reports. Neurosurgery 1997;41(04):951-955; discussion 955-956

12 Marshman LA, Benjamin JC, Chawda SJ, David KM. Acute obstructive hydrocephalus associated with infratentorial subdural hygromas complicating Chiari malformation Type I decompression. Report of two cases and literature review. J Neurosurg 2005;103(04):752-755

13 Stavrinos NG, Taylor R, Rowe A, Whittle IR. Posterior fossa surgery complicated by a pseudomeningocele, bilateral subdural hygromata and cerebellar cognitive affective syndrome. $\mathrm{Br} \mathrm{J}$ Neurosurg 2008;22(01):107-109

14 Filis AK, Moon K, Cohen AR. Symptomatic Subdural Hygroma and Hydrocephalus following Chiari I Decompression. Pediatr Neurosurg 2009;45(06):425-428

15 Suzuki F, Kitagawa T, Nozaki K, Takagi K. Subacute subdural hygroma and presyrinx formation after foramen magnum decompression with duraplasty for Chiari type 1 malformation. Neurol Med Chir (Tokyo) 2011;51(05):389-393

16 Bahuleyan B, Menon G, Hariharan E, Sharma M, Nair S. Symptomatic posterior fossa and supratentorial subdural hygromas as a rare complication following foramen magnum decompression for Chiari malformation Type I. J Neurosurg 2011;114(02):510-513 